\title{
La réforme de la loi du travail au Brésil
}

\author{
Juliano Barra
}

\section{(2) OpenEdition}

\section{Journals}

Édition électronique

URL : https://journals.openedition.org/rdctss/2045

DOI : $10.4000 /$ rdctss.2045

ISSN : 2262-9815

Éditeur

Centre de droit comparé du travail et de la sécurité sociale

Édition imprimée

Date de publication : 1 avril 2018

Pagination : 128-131

ISSN : 2117-4350

\section{Référence électronique}

Juliano Barra, «La réforme de la loi du travail au Brésil », Revue de droit comparé du travail et de la sécurité sociale [En ligne], 1 | 2018, mis en ligne le 01 novembre 2021, consulté le 12 novembre 2021. URL : http://journals.openedition.org/rdctss/2045; DOI : https://doi.org/10.4000/rdctss.2045

\section{(c) (i) (9)}

Revue de droit comparé du travail et de la sécurité sociale est mise à disposition selon les termes de la Licence Creative Commons Attribution - Pas d'Utilisation Commerciale - Pas de Modification 4.0 International. 


\section{LA RÉFORME DE LA LOI DU TRAVAIL AU BRÉSIL}

La «Consolidation des lois du travail » (ci-après CLT) est le texte le plus fondamental du droit du travail brésilien. Publiée le $1^{\text {er }}$ mai 1943 et comptant plus de 900 articles, la CLT est semblable dans sa forme aux codes du travail des pays de l'Europe continentale. Ce texte régule la part la plus importante des relations de travail au Brésil et a été, pendant plusieurs années, une réponse juridique apportée aux questions des relations de travail, et ce, à la lumière des textes internationaux. Cela a été, et est encore, le texte de référence par excellence du droit du travail brésilien. 70 ans après sa promulgation, le texte a connu des vicissitudes et est, en grande partie, devenu obsolète. Avec la volonté de dynamiser et moderniser les relations de travail, le législateur vient d'adopter la plus importante réforme du droit du travail brésilien avec la loi n 13467 du 13 juillet 2017, modifiant une centaine d'articles de la CLT. Entrées en vigueur depuis novembre 2017, les modifications portent sur les relations individuelles (I) et collectives de travail (II).

\section{I - LA RÉFORME DE LA CLT ET LES RELATIONS INDIVIDUELLES DU TRAVAIL}

La réforme de la CLT concerne une multitude de dispositions. On abordera dans le cadre de ce commentaire la mise en place d'un barème des indemnités octroyées en cas de dommages extrapatrimoniaux subis par les salariés $(A)$ et la régulation des «zero-hour contracts » à la brésilienne (B).

\section{A. UN BARÈME D'INDEMNITÉS DES DOMMAGES EXTRAPATRIMONIAUX}

La loi du 13 juillet 2017 a introduit la barémisation des indemnités en cas de condamnation par le juge du travail brésilien pour dommages extrapatrimoniaux. Le nouvel article 223-B de la CLT qualifie de dommage extrapatrimonial un acte ou une omission qui porte atteinte à la sphère morale ou existentielle du salarié. Il s'agit aussi de dommages commis contre son honneur, son image, sa vie privée, sa liberté d'action, sa sexualité, sa santé, sa nationalité, son ethnie ou son intégrité physique.

Pour calculer l'indemnisation due au salarié, les juges devront vérifier tout d'abord l'intensité du dommage ou la douleur subie, ensuite les conséquences personnelles et sociales du dommage, son extension et sa durée, l'existence ou non d'une faute et, enfin, la situation sociale et économique des parties au litige. Une fois vérifié tout cela, s'appliquera l'article 223-G §1 de la CLT qui prévoit les plafonds des indemnités: en cas d'une infraction de nature légère, l'indemnité pourra atteindre jusqu'à trois fois la valeur du plafond des prestations versées par le régime général de sécurité sociale; pour une infraction de nature moyenne, jusqu'à cinq fois le montant et pour une infraction grave, jusqu'à vingt fois la valeur du même plafond; pour une infraction lourde, l'indemnité pourra aller jusqu'à cinquante fois la valeur du plafond. Par exemple, actuellement la valeur du plafond des prestations de sécurité sociale est de 564580 Reais, (environ 1411 euros). En cas d'un 
dommage de nature légère, le juge pourra imposer une indemnité maximale à l'employer de 16935 Reais (environ 4200 euros); dans le cas de dommage lourd, la valeur pourra aller jusqu'à 282290 Reais (environ 70000 euros).

En revanche, ce barème n'est pas applicable en cas de dommages résultant de décès; tel est le cas d'une indemnité versée en raison des dommages moraux causés aux ayants droit d'un salarié décédé d'un accident de travail. Cette barémisation a pour but de donner plus de prévisibilité à l'employeur et d'éviter des indemnisations très disparates dans ce pays de taille continentale. La réforme comporte aussi une importante innovation en matière de contrats de travail intermittents.

\section{B - LA MISE EN ÆUVRE DE CONTRATS INTERMITTENTS}

Le nouveau $\S 3$ de l'article 443 de la CLT prévoit la mise en place d'une sorte de «zerohour contracts » à la brésilienne, nouvelle forme de travail intermittent. Ainsi, est qualifié intermittent le contrat de travail dont la prestation de services n'est pas continue, réalisé par alternance en heures, jours ou mois, indépendamment du type d'activité du salarié ou de l'employeur, à l'exception des aéronautes, régis par leur propre loi. L'élément de subordination est toujours présent afin d'accorder, malgré la précarité, le caractère d'une relation de travail salarié. En peu de mots, il s'agit d'un contrat à durée indéterminée destiné à pourvoir un emploi permanent comportant par nature une alternance de périodes travaillées et de périodes non travaillées. II n'est pas nécessaire que ce type de contrat ait comme source un accord ou convention collective de travail; mais la loi permet que les partenaires sociaux négocient sur ce sujet.

Ce contrat doit être conclu par écrit et contenir l'identification des parties, le taux de I'heure ou d'une journée de travail, le lieu de travail et la périodicité du versement de la rémunération. L'employeur enverra au salarié, avec un préavis d'au moins trois jours, une convocation l'informant du jour et des heures de la prestation de travail. Le salarié aura vingtquatre heures pour y répondre, son silence valant refus de la mission. Le refus du salarié n'a pas pour effet d'exclure le caractère subordonné de la relation. Une fois acceptée l'offre de travail, la partie qui l'enfreint sans raison valable paiera à l'autre partie, dans les trente jours, une amende de $50 \%$ de la valeur de la rémunération due. Le $\S 5$ du nouvel article 452-A de la CLT dispose que la période d'inactivité ne doit pas être considérée comme du temps à disposition de l'employeur, le salarié pouvant se mettre au service d'autres employeurs. Pour chaque période de douze mois le salarié acquiert le droit de jouir, durant les douze mois suivants, d'un mois de congé payé, au cours duquel il ne pourra pas être sollicité pour travailler chez le même employeur.

Reste à noter quaprès une période de 12 mois sans sollicitation du salarié par l'employeur, le contrat de travail intermittent sera considéré comme résilié, l'article 452-E de la CLT prévoyant les indemnités légales applicables à cette situation. Afin d'éviter des fraudes, jusqu'au 31 décembre 2020, des salariés licenciés liés par des contrats de travail à durée indéterminée ne pourront pas être employés en vertu d'un contrat de travail intermittent par le même employeur qu'après le délai de dix-huit mois, à compter de la date du licenciement.

La doctrine brésilienne est très critique à l'égard de cette nouvelle forme d'emploi en raison de l'insécurité juridique pesant non seulement sur le salarié, mais aussi sur les employeurs qui prendront des risques avec des contrats intermittents. La Cour suprême a été saisie (Supremo Tribunal Federal - STF) par une fédération de syndicat de salariés 
d'une action en inconstitutionnalité (ADI 5829) mettant en avant que ce contrat intermittent précarise les relations de travail en autorisant des rémunérations en dessous du salaire minimum, et qu'il existerait ainsi une violation du principe d'égalité et une régression des droits sociaux (théorie de l'effet cliquet). À l'heure actuelle, le futur du "zero-hour contract» à la brésilienne n'est pas assuré. La réforme de la CLT a également impacté les relations collectives du travail.

\section{II - LA RÉFORME DE LA CLT ET LES RELATIONS COLLECTIVES DU TRAVAIL}

Le droit des relations collectives du travail au Brésil a été fortement bouleversé. On peut même se demander si la Constitution et les Conventions internationales de I'OIT portant sur la négociation collective ne sont pas violées par cette réforme. Il est possible de retenir l'existence d'une forme de "flexsécurisation » des effets de la négociation collective (A), tandis que les accords collectifs d'entreprise sont en passe de prévaloir sur les conventions collectives de branche (B).

\section{A - DE LA « FLEXSÉCURISATION 》 DES EFFETS DE LA NÉGOCIATION COLLECTIVE}

Il est un élément qui bouleversera considérablement la routine des syndicats et des entreprises au Brésil, c'est le fait qu'en cas de conflit entre une accord/convention collective de travail et la loi, ce sont les premiers qui devront prévaloir, que leur contenu soit plus favorable ou non. Désormais, les accords et conventions collectives de travail prévalent sur les dispositions légales, même si ces dernières comportent des prévisions plus favorables aux salariés. Cette touche de «flexsécurisation » prévu dans l'article 611-A de la CLT donne des garanties pour que les entreprises et les partenaires sociaux puissent négocier avec plus de sécurité juridique, évitant que certaines modalités préalablement négociées soient annulées par la justice quelques années après.

Néanmoins, cette prévalence de l'accord collectif sur la loi n'est pas absolue. Premièrement, rappelons que la Constitution du Brésil est très analytique et qu'elle comprend spécialement l'article 7 doté de 34 paragraphes qui représentent un seuil de protection constitutionnel de divers droits, comme par exemple la protection contre le licenciement arbitraire ou sans motif légitime ${ }^{1}$. Deuxièmement, le législateur a établi dans le nouvel article 611-B de la CLT un ensemble de droits qui ne pourront pas être minorés

1 Les principaux droits protégés par l'article 7 de la Constitution sont: la protection contre le licenciement arbitraire ou sans motif légitime; la garantie de l'assurance-chômage en cas de chômage involontaire; l'institution d'un "fonds de garantie»; l'intangibilité du salaire, sauf convention ou accord collectif contraire; droit au treizième mois; le droit à une sur rémunération du travail de nuit; le droit à la participation aux bénéfices de l'entreprise, et à la participation à la gestion de l'entreprise, selon des modalités définies par la loi ; le droit à une durée de travail n'excédant pas 8 heures par jour et 44 heures par semaine, sauf exceptions; le droit à un repos hebdomadaire rémunéré, de préférence le dimanche; le droit à la rémunération des heures supplémentaires à un taux majoré d'au moins $50 \%$; le droit à un congé payé annuel assorti d'une majoration d'au moins un tiers de la rémunération; le droit des femmes à la promotion sur le marché du travail conformément à la loi; le droit à un préavis proportionnel à l'ancienneté, et d'au moins 30 jours; la reconnaissance des conventions et accords collectifs du travail; le droit à l'assurance contre les accidents du travail à la charge de l'employeur; l'interdiction de toute discrimination, en matière de salaire ou d'embauche, à l'égard des travailleurs handicapés. 
ou supprimés par un accord collectif de travail, comme le salaire minimum, le paiement majoré de $50 \%$ des heures supplémentaires ou encore les dispositions sur le droit de grève. Nonobstant la réforme, un ordre public social est encore applicable et reste un instrument essentiel de protection des salariés. La réforme a également mis en œuvre des limites au « principe de faveur » avec la prévalence des accords d'entreprise.

\section{B - LA PRIMAUTÉ DES ACCORDS D'ENTREPRISE}

Il importe d'observer qu'aux termes de la CLT les partenaires sociaux ont compétence pour établir des accords ou conventions collectifs. L'article 611 CLT prévoit par ailleurs que la convention collective est un accord à caractère normatif, conclu entre organisations professionnelles, c'est-à-dire entre une organisation patronale et une organisation syndicale au moins, représentant les catégories concernées par la convention, et réglementant les relations individuelles de travail. Le $\S 1$ de cet article habilite également les syndicats représentant les catégories professionnelles concernées à conclure des accords collectifs, qui se caractérisent par le fait qu'ils sont négociés directement avec l'entreprise réglementant les conditions de travail applicables dans le cadre de l'entreprise.

En matière de négociation collective, la rédaction originale de l'article 620 de la CLT énonçait une sorte de « principe de faveur » prévoyant que les stipulations d'une convention collective de branche, lorsqu'elles sont plus favorables, l'emportent sur celles prévues dans un accord collectif d'entreprise ou dans le contrat de travail. Toutefois, cette hiérarchie a été totalement bouleversée. Depuis novembre 2017, l'article 620 prévoit que les conditions établies dans les accords collectifs d'entreprise prévaudront sur celles prévues dans les conventions collectives de branche y compris en cas de dispositions plus favorables. Le droit des relations collectives du travail au Brésil place au centre de la négociation collective les conditions de travail dans l'entreprise par rapport à la branche. La loi est claire sur ce point: l'accord d'entreprise doit toujours prévaloir sur l'accord de branche.

Enfin, la CLT a su, moyennant une adaptation de son contenu, traverser le temps et faire face aux évolutions considérables de la société brésilienne. La toute récente et plus importante réforme du droit du travail démontre que sa centralité est encore d'actualité, mais non sans critiques et sans contentieux (la Cour Suprême ayant été saisie de plusieurs actions en inconstitutionnalité mettant en cause la loi du 13 juillet 2017). 